\title{
ANALYTIC DISCS, PLURISUBHARMONIC HULLS, AND NON-COMPACTNESS OF THE $\bar{\partial}$-NEUMANN OPERATOR
}

\author{
SÖNMEZ ŞAHUTOĞLU AND EMIL J. STRAUBE
}

\begin{abstract}
We show that a complex manifold $M$ in the boundary of a smooth bounded pseudoconvex domain $\Omega$ in $\mathbb{C}^{n}$ is an obstruction to compactness of the $\bar{\partial}$-Neumann operator on $\Omega$, provided that at some point of $M$, the Levi form of $b \Omega$ has the maximal possible rank $n-1-\operatorname{dim}(M)$ (i.e. the boundary is strictly pseudoconvex in the directions transverse to $M$ ). In particular, an analytic disc is an obstruction, provided that at some point of the disc, the Levi form has only one zero eigenvalue (i.e. the eigenvalue zero has multiplicity one). We also show that a boundary point where the Levi form has only one zero eigenvalue can be picked up by the plurisubharmonic hull of a set only via an analytic disc in the boundary.
\end{abstract}

\section{INTRODUCTION AND RESULTS}

Let $\Omega$ be a smooth bounded pseudoconvex domain in $\mathbb{C}^{n}$. The $\bar{\partial}$-Neumann operator $N$ is the inverse of the complex Laplacian $\square=\overline{\partial \partial}^{*}+\bar{\partial}^{*} \bar{\partial}$ on square integrable $(0,1)$ forms on $\Omega$. Its regularity theory plays an important role both in partial differential equations and in several complex variables; detailed information can be found in [5, 10, 14. In particular, the question of when $N$ is, or is not, compact is of interest in various contexts, among them global regularity (24, 5]), Toeplitz operators ([17] and its references), semiclassical analysis of Schrödinger operators ([18, 12]), and kernels solving $\bar{\partial}([21])$. An important motivation for studying compactness has emerged over the last ten years, as it became increasingly clear how subtle the (a priori more important) property of global regularity is (see the surveys [5, 11]): compactness is more robust (it localizes, for example) and thus should be more readily amenable to a characterization in terms of properties of the boundary.

There is a fairly general potential theoretic condition on the boundary of a domain which implies that the $\bar{\partial}$-Neumann operator is compact. This condition was introduced, and shown to imply compactness, by Catlin([9]) under the name of prop$\operatorname{erty}(\mathrm{P})$. A detailed study of this property, from the point of view of the Choquet theory of the cone of plurisubharmonic functions, can be found in [31. In particular, the boundary of a smooth bounded pseudoconvex domain satisfies property $(\mathrm{P})$ if and only if continuous functions on the boundary can be approximated uniformly (on the boundary) by functions continuous on the closure and plurisubharmonic in the interior (31], théorème 2.1). For a discussion of property $(\mathrm{P})$ in the spirit of Oka's lemma and related results, see [20]. McNeal showed that a relaxed version of $\operatorname{property}(\mathrm{P})$ still implies compactness ([27]). An interesting sufficient condition for compactness,

2000 Mathematics Subject Classification: 32W05.

Research supported in part by NSF grant number DMS-0100517. 
related to both property $(\mathrm{P})$ and the relaxed version in [27], was given in [34. Recently, the second author introduced a more geometric approach to compactness of the $\bar{\partial}$-Neumann operator on domains in $\left.\mathbb{C}^{2}(32]\right)$.

The most blatant violation of propertiy $(\mathrm{P})$ as well as of the sufficient conditions in [34, [27, and 32] is an analytic disc in the boundary. Such discs also cause failure of hypoellipticity for $\bar{\partial}[\mathbf{8}, 13]$ ). The question whether they also cause failure of compactness is thus a natural one. That the answer is yes in the case of domains in $\mathbb{C}^{2}$ is an unpublished result of Catlin from the early eighties; a proof for the case of domains with Lipschitz boundary is in [17. (Note that the converse is false: there are obstructions to compactness of the $\bar{\partial}$-Neumann operator considerably more subtle than discs in the boundary, see [26, 17].) The situation is completely understood in locally convexifiable domains: $N$ is compact (if and) only if the boundary contains no analytic disc $(16,17)$. It is folklore that the methods that work in $\mathbb{C}^{2}$ also show that, in any dimension $n$, if the $\bar{\partial}$-Neumann operator is compact, then the boundary cannot contain an $(n-1)$-dimensional complex manifold. However, whether a disc is necessarily an obstruction to compactness is open. We show in this paper that this is the case when the disc contains a point at which the boundary is strictly pseudoconvex in the directions transverse to the disc. More generally, one can trade one strictly positive eigenvalue of the Levi form for an increase of one in the dimension of the complex manifold that is to obstruct compactness.

Although we are mainly interested in compactness of the $\bar{\partial}$-Neumann operator $N$, our arguments proceed via existence of a compact solution operator to $\bar{\partial}$ on $(0,1)$-forms. A solution operator $T$ for $\bar{\partial}$ on $(0,1)$-forms is a (continuous) operator $T: L_{(0,1)}^{2}(\Omega) \cap \operatorname{ker}(\bar{\partial}) \rightarrow L^{2}(\Omega)$ such that $\bar{\partial} T(\alpha)=\alpha$ for all $\alpha \in L_{(0,1)}^{2}(\Omega) \cap \operatorname{ker}(\bar{\partial})$. It is well known that if $N$ is compact, then the canonical solution operator $\bar{\partial}^{*} N$ is compact (considerably more can be said, see Lemma 1.1 in [17]). Note that since the projection of $L^{2}(\Omega)$ onto the orthogonal complement of the holomorphic functions preserves compactness, saying that there exists a compact solution operator for $\bar{\partial}$ is the same as saying that the canonical solution operator is compact.

Theorem 1. Let $\Omega$ be a smooth bounded pseudoconvex domain in $\mathbb{C}^{n}, n \geq 2$. Let $P \in b \Omega$ and assume that the Levi form of $b \Omega$ at $P$ has the eigenvalue zero with multiplicity at most $k, 1 \leq k \leq n-1$. If there exists a compact solution operator for $\bar{\partial}$ on $(0,1)$-forms (in particular, if the $\bar{\partial}$-Neumann operator on $\Omega$ is compact), then $b \Omega$ does not contain a $k$-dimensional complex manifold through $P$.

In particular, if the Levi form of the boundary has at most one degenerate eigenvalue at all boundary points, then compactness of the $\bar{\partial}$-Neumann operator implies that there are no discs in the boundary. A special case of this occurs imlicitly in [23], Theorem 1.1, for domains fibered over a Reinhardt domain in $\mathbb{C}^{2}$. We will give the proof of Theorem 1 in section 2, along with the proof of the next corollary. Note that when the set of Levi flat boundary points has nonempty (relative) interior, then it is foliated by $(n-1)$-dimensional complex manifolds. By the folklore result mentioned above, this is incompatible with compactness of the $\bar{\partial}$-Neumann operator. It is an easy consequence of Theorem 1 that this result holds for the (in general much bigger) set of weakly pseudoconvex points. 
Corollary 1. Let $\Omega$ be a smooth bounded pseudoconvex domain in $\mathbb{C}^{n}, n \geq 2$. If there exists a compact solution operator for $\bar{\partial}$ on $(0,1)$-forms (in particular, if the $\bar{\partial}$-Neumann operator on $\Omega$ is compact), then the set of weakly pseudoconvex points in $b \Omega$ has empty (relative) interior.

Remark 1. If $b \Omega$ is not strictly pseudoconvex in the directions transverse to the complex submanifold at $P$, then the boundary is 'more flat'. In general, one would expect this situation to be even more favorable to noncompactness of the $\bar{\partial}$-Neumann operator. However, our present methods do not give this.

Denote by $P(\bar{\Omega})$ the set of plurisubharmonic functions on $\Omega$ that are continuous up to the boundary of $\Omega$. Define the plurisubharmonic hull, $\widehat{K}$, of a compact set $K \subset \bar{\Omega}$ as follows:

$$
\widehat{K}=\left\{z \in \bar{\Omega}: f(z) \leq \sup _{w \in K} f(w) \text { for all } f \in P(\bar{\Omega})\right\}
$$

The same hull results when taken with respect to any of the following sets of functions: plurisubharmonic functions smooth up to the boundary, holomorphic functions smooth up to the boundary, or holomorphic functions continuous up to the boundary (see for example [6, 7, 19]). The intersection of $\widehat{K}$ with the boundary is determined by $K \cap b \Omega: \widehat{K} \cap b \Omega=(\widehat{K \cap b \Omega}) \cap b \Omega$ ([7], Theorem 3.1.7, [19] Proposition 2). Thus a compact subset $K$ of $\bar{\Omega}$ which picks up hull in the boundary, that is, where $\widehat{K} \cap b \Omega \neq K \cap b \Omega$, represents an obvious violation of property $(\mathrm{P})$ (the situation with respect to the conditions in [34, [27], and 32] seems not to be understood at present) as well as an obstruction to hypoellipticity of $\bar{\partial}$ ( $[8]$, Theorem 3). Such sets therefore present themselves as interesting candidates for obstructions to compactness in the $\bar{\partial}$-Neumann problem. In $\mathbb{C}^{2}$, this leads to nothing new: a compact $K$ can pick up hull in the boundary only via an analytic disc contained in the boundary ([8], Theorem 4). In higher dimensions, however, sets can pick up hull in the boundary in less obvious ways, that is, even when the boundary contains no analytic disc ([8], Theorem 5). Thus, in view of the case $k=1$ of Theorem 11, the question arises how a point in the boundary can be picked up by the plurisubharmonic hull if the Levi form at the point has only one degenerate eigenvalue. Perhaps not surprisingly, the situation is the same as in $\mathbb{C}^{2}$ : there has to be an analytic disc in the boundary through the point.

Proposition 1. Let $\Omega$ be a smooth bounded pseudoconvex domain in $\mathbb{C}^{n}, n \geq 2$. Let $P$ be a boundary point where the Levi form of $b \Omega$ has at most one degenerate eigenvalue. Then there is a compact subset $K$ of $\bar{\Omega}$ with $P \in \widehat{K} \backslash K$ if and only if $b \Omega$ contains an analytic disc through $P$.

Proposition 1 can be shown along the lines of the proof for the $\mathbb{C}^{2}$ case given in [6], but some additional work is needed. We will give the details in section 3 .

Remark 2. Sibony has characterized the domains for which $\widehat{K} \cap b \Omega=K \cap b \Omega$ for all compact subsets $K$ of $\bar{\Omega}$ by hypoellipticity of $\bar{\partial}$ in local $L^{2}$-spaces: for every $\bar{\partial}$ closed $(0,1)$-form $\alpha$ with locally square integrable coefficients in $\Omega$ there should exist a locally square integrable function $u$ on $\Omega$ solving $\bar{\partial} u=\alpha$ and such that the singular support of $u$ in $\bar{\Omega}$ is equal to the singular support of $\alpha$ ([30]). Combining this with 
Theorem 1 and Proposition 1 above shows that on a smooth bounded pseudoconvex domain, if the Levi form has at most one degenerate eigenvalue at each boundary point, compactness of the $\bar{\partial}$-Neumann operator implies hypoellipticity of $\bar{\partial}$ in the space of locally square integrable forms. It would be very interesting to have a direct proof of this implication.

\section{Proofs of Theorem 1 and Corollary 1}

We will need to control the boundary geometry near a point of a complex manifold in the boundary. This is accomplished in the following lemma.

Lemma 1. Let $\Omega$ be a smooth bounded pseudoconvex domain in $\mathbb{C}^{n}, M$ a complex manifold of dimension $k$ in $b \Omega$, and $P \in M$. Then there is a ball $B$ centered at $P, a$ biholomorphic map $G: B \rightarrow G(B)$ such that

(i) $G(P)=0$

(ii) $G(M \cap B)=\left\{w \in G(B) \mid w_{k+1}=\cdots=w_{n}=0\right\}$

(iii) the real normal to $G(b \Omega \cap B)$ at points of $G(M \cap B)$ is given by the $\operatorname{Re}\left(w_{n}\right)$-axis.

In other words, there is a local holomorphic change of coordinates so that in the new coordinates, $M$ is affine and the (real) unit normal to the boundary is constant along $M$.

To prove the lemma, note first that we can change coordinates near $P$ so that $M$ is locally given by $\left\{z \mid z_{k+1}=\cdots=z_{n}=0\right\}$, and $P=0$. In these coordinates, let $\rho$ be a defining function for $b \Omega$ near 0 . We may assume that $\partial \rho / \partial z_{n} \neq 0$. There exists a real-valued $C^{\infty}$ function $h$ in a neighborhood of 0 such that the normal $e^{h}\left(0, \cdots, 0, \frac{\partial \rho}{\partial \bar{z}_{k+1}}, \cdots, \frac{\partial \rho}{\partial \bar{z}_{n}}\right)$ is conjugate holomorphic on $M$ (each component is conjugate holomorphic). When $M$ is a disc, this is the conclusion of Lemma 1 in [1]. One can adapt these methods to cover the case where $\operatorname{dim} M>1$. Alternatively, the statement is a special case of the theorem in [33], specifically the equivalence of $(i i)$ and $(i v)$. Note that the form $\alpha$ appearing in $(i v)$ of that theorem is real, and its restriction to $M$ is closed by the lemma in section 2 of [4]. Therefore, there is a real valued function $h$ such that $d(-h)=\alpha$ on $M$ (near 0 ). The proof of $(i i) \Leftrightarrow(i v)$ in 33] shows that any real-valued $C^{\infty}$ extension of $h$ to a full neighborhood of 0 will do.

We now define a biholomorphic change of coordinates (near 0 ) by $\widehat{G}\left(z_{1}, \cdots, z_{n}\right)=$ $\left(z_{1}, \cdots, z_{n}\right)+S(z)$, where

$$
S(z)=\left(0, \cdots, 0, \sum_{j=k+1}^{n} z_{j} e^{h\left(z_{1}, \cdots, z_{k}, 0, \cdots, 0\right)} \frac{\partial \rho}{\partial z_{j}}\left(z_{1}, \cdots, z_{k}, 0, \cdots, 0\right)\right) .
$$

Then $\widehat{G}(M) \subset\left\{z_{k+1}=\cdots z_{n}=0\right\}$, and at points of $M$, the complex derivative of $\widehat{G}$ maps the complex tangent space to $b \Omega$ onto the complex hypersurface $\left\{z_{n}=0\right\}$. Consequently, in these new coordinates ( which we again denote by $\left(z_{1}, \cdots, z_{n}\right)$ ), $M$ is of the same form as before, but the complex tangent space to $b \Omega$ is constant, namely it is the hyperplane $\left\{z_{n}=0\right\}$. Consider now the real unit normal to $b \Omega$. Its restriction to $M$ is of the form $\left(0, \cdots, 0, e^{i \theta}\right)$. Using Lemma 1 from [1] once more shows that the function $\theta$ is harmonic on each disc in $M$, i.e. it is pluriharmonic on $M$. Denote by $h_{1}$ a pluriharmonic conjugate. The final coordinate change

$$
\left(z_{1}, \cdots, z_{n}\right) \rightarrow\left(z_{1}, \cdots, z_{n-1}, z_{n} e^{-h_{1}\left(z_{1}, \cdots, z_{k}, 0, \cdots, 0\right)-i \theta\left(z_{1}, \cdots, z_{k}, 0, \cdots, 0\right)}\right)
$$


rotates the real normal so that the unit normal becomes constant on $M$. Combining the three local biholomorphic coordinate changes gives the map $G$ with the properties required in Lemma 1 .

The proof of Theorem 1 will be indirect; we will show that if the boundary contains a $k$-dimensional complex manifold through $P$, then the $\bar{\partial}$-Neumann operator on $\Omega$ is not compact. The source of noncompactness is contained in the following lemma. Denote by $A(\Omega)$ the Bergman space of a domain $\Omega$ in $\mathbb{C}^{n}$, that is, the closed subspace of $L^{2}(\Omega)$ consisting of holomorphic functions.

Lemma 2. Let $\Omega$ be a bounded pseudoconvex domain in $\mathbb{C}^{n}$, smooth near the strictly pseudoconvex boundary point $P$. Assume the pseudoconvex domain $\Omega_{1}$ is contained in $\Omega$, shares the boundary point $P$, and is smooth near $P$. Then the restriction operator from $A(\Omega)$ to $A\left(\Omega_{1}\right)$ is not compact.

We prove the lemma by constructing a sequence of norm 1 functions in $A(\Omega)$ which has no convergent subsequence in $A\left(\Omega_{1}\right)$. Let $\left\{P_{j}\right\}_{j=1}^{\infty}$ be a sequence of points on the common interior normal to $b \Omega$ and $b \Omega_{1}$ at $P$ such that $\lim _{j \rightarrow \infty} P_{j}=P$. Set $f_{j}(z)=K_{\Omega}\left(z, P_{j}\right) / K_{\Omega}\left(P_{j}, P_{j}\right)^{1 / 2}$, where $K_{\Omega}$ denotes the Bergman kernel of $\Omega$. The normalization is so that $\left\|f_{j}\right\|_{L^{2}(\Omega)}=1$ for all $j$. For $z \in \Omega$ fixed, the function $K_{\Omega}(z, \cdot)$ is smooth up to the boundary near $P$ (in fact, the subelliptic estimates for the $\bar{\partial}$ Neumann problem near $P$ have considerably stronger consequences for the kernel function [3], 2]), and $K_{\Omega}\left(P_{j}, P_{j}\right) \rightarrow \infty$ as $j \rightarrow \infty$ (see e.g. [22], Theorem 3.5.1). Consequently, $f_{j}(z) \rightarrow 0$ for all $z \in \Omega$. On the other hand,

$$
\left\|f_{j}\right\|_{L^{2}\left(\Omega_{1}\right)}^{2}=\frac{\left\|K_{\Omega}\left(\cdot, P_{j}\right)\right\|_{L^{2}\left(\Omega_{1}\right)}^{2}}{K_{\Omega}\left(P_{j}, P_{j}\right)} \geq \frac{K_{\Omega}\left(P_{j}, P_{j}\right)}{K_{\Omega_{1}}\left(P_{j}, P_{j}\right)} \geq C>0 .
$$

The first inequality follows by applying the reproducing property of $K_{\Omega_{1}}$ to the function $K_{\Omega}\left(\cdot, P_{j}\right)$ viewed as an element of $A\left(\Omega_{1}\right)$. (This argument comes verbatim from [16], p. 637.) Because both $\Omega$ and $\Omega_{1}$ are strictly pseudoconvex at $P$, the kernel asymptotics at $P$ are the same ([22], Theorem 3.5.1), and the second inequality follows. We conclude that $\left\{f_{j}\right\}_{j=1}^{\infty}$ has no subsequence that converges in $A\left(\Omega_{1}\right)$, and the proof of Lemma 2 is complete.

To prove Theorem 11, we use the simple geometry of $b \Omega$ in the new coordinates (near $P)$ to construct a sequence of $\bar{\partial}$-closed $(0,1)$-forms that will lead to a contradiction when combined with the existence of a compact solution operator for $\bar{\partial}$ on $\Omega$. The argument follows very closely [16, section 4, and [17, proof of Proposition 4.1. In turn, those arguments draw substantially on ideas from [8] and [13. We keep the notation from Lemma 1. Denote $G(B \cap \Omega)$ by $\widetilde{\Omega}$. There exist open neighborhoods $V_{2} \subset \subset V_{1} \subset \subset V_{0} \subset \subset G(B)$ of $P=0$ in $\mathbb{C}^{n}$ and a small enough ball $\widetilde{B}$ in $\mathbb{C}^{n-k}$ such that $M_{0} \times \widetilde{B} \subset \widetilde{\Omega}$, where we set $M_{j}=M \cap V_{j}, j=0,1,2$. Here we have used that the real normal to the boundary is constant along $M$. Let $S_{0}$ be the slice of $V_{0} \cap \widetilde{\Omega}$ in $\mathbb{C}^{n-k}$ that is perpendicular to $M$. Because the rank of the Levi form is preserved under biholomorphisms, $S_{0}$ is strictly pseudoconvex at 0 . Lemma 2 shows that there exists a sequence of holomorphic functions $\left\{f_{j}\right\}_{j=1}^{\infty}$ that is bounded in $A\left(S_{0}\right)$ and has no convergent subsequence in $A(\widetilde{B})$. Using the Ohsawa-Takegoshi extension theorem [28] one can extend $f_{j}$ from $S_{0}$ to $\widetilde{\Omega}$ for each $j$ to get a bounded 
sequence of holomorphic functions $\left\{F_{j}\right\}_{j=1}^{\infty}$ in $A(\widetilde{\Omega})$. Define $\beta_{k}(z)=F_{k}(z) \chi(z)$ where $\chi: V_{0} \rightarrow[0,1]$ is a smooth function such that

$$
\chi(z)=\left\{\begin{array}{lll}
1 & \text { if } & z \in M_{2} \times \widetilde{B} \\
0 & \text { if } & z \notin V_{1}
\end{array}\right.
$$

Let $\alpha_{k}(z)=\bar{\partial}\left(\chi(z) F_{k}(z)\right)=F_{k}(z) \bar{\partial} \chi(z) .\left\{\alpha_{k}\right\}_{k=1}^{\infty}$ is a bounded sequence in $L_{(0,1)}^{2}(\widetilde{\Omega})$. We pull $\alpha_{k}$ back via $G$ and view the resulting form $\widetilde{\alpha_{k}}$ as a $\bar{\partial}$-closed form in $\Omega$ (by extending by 0 outside $B$ ). The sequence $\left\{\widetilde{\alpha_{k}}\right\}_{k=1}^{\infty}$ is bounded in $L_{(0,1)}^{2}(\Omega)$. By assumption, there is a compact solution operator for $\bar{\partial}$ on $\Omega$. Applying this solution operator to the sequence $\left\{\widetilde{\alpha_{k}}\right\}_{k=1}^{\infty}$ and passing to a subsequence if necessary yields a sequence $\left\{\widetilde{g_{k}}\right\}_{k=1}^{\infty}$ which converges in $L^{2}(\Omega)$ such that for all $k$, we have $\bar{\partial} \widetilde{g_{k}}=\widetilde{\alpha_{k}}$. Restricting to $B \cap \Omega$ and pushing forward to $\widetilde{\Omega}$, gives a sequence $\left\{g_{k}\right\}_{k=1}^{\infty}$ that converges in $L^{2}(\widetilde{\Omega})$ and such that for all $k, \bar{\partial} g_{k}=\alpha_{k}$. Finally, setting $h_{k}(z)=g_{k}(z)-\chi(z) F_{k}(z)$ gives a sequence of holomorphic functions on $\widetilde{\Omega}$. Notice that $h_{k}=g_{k}$ on $\widetilde{\Omega} \backslash V_{1}$, so $\left\{h_{k}\right\}_{k=1}^{\infty}$ converges in $L^{2}\left(\widetilde{\Omega} \backslash \overline{V_{1}}\right)$. The $L^{2}$-norm of a holomorphic function on a spherical shell dominates the $L^{2}$-norm of the function on the whole ball. Combining this observation with Fubini's theorem implies that $\left\{h_{k}\right\}_{k=1}^{\infty}$ converges in $L^{2}\left(M_{2} \times \widetilde{B}\right)$. Hence so does $\left\{F_{k}\right\}_{k=1}^{\infty}$ (since $\left\{g_{k}\right\}_{k=1}^{\infty}$ also converges there and $\chi \equiv 1$ on $M_{2} \times$ $\widetilde{B})$. Using the submean value property on balls in the variables $w_{1}, w_{2}, \cdots, w_{k}$ while

$\left(w_{k+1}, \cdots, w_{n}\right) \in \widetilde{B}$ stays fixed and then again Fubini's theorem we get that $\left\{f_{k}\right\}_{k=1}^{\infty}$ converges in $A(\widetilde{B})$. This is the contradiction we seek. The proof of Theorem [1] is therefore complete.

We next prove Corollary 1, Let $V$ be a nonempty open subset of $b \Omega$ contained in the set of weakly pseudoconvex points. Define $m$ to be the maximum rank of the Levi form on $V$ and let $P \in V$ be a point where the Levi form has rank $m$ (such a point exists, since the rank assumes only finitely many values on $V$ ). Then near $P$, the rank is at least $m$, hence equal to $m$. Therefore, $b \Omega$ is foliated, near $P$, by complex manifolds of dimension $n-1-m$ (see for example [15]). Theorem 1] (for $k=n-1-m)$ implies that the $\bar{\partial}$-Neumann operator on $\Omega$ is not compact. This contradicts the assumption in Corollary 1 .

\section{Proof of Proposition 1}

We denote the Levi form of $b \Omega$ by $L(Y, \bar{Z})$ for complex tangential vector fields $Y, Z$ of type $(1,0)$. We similarly use the notation $L_{f}(Y, \bar{Z})$ for the full complex Hessian of a function $f$. Let $J$ denote the complex structure map of (the tangent bundle of) $\mathbb{C}^{n}$, $F_{A}^{t}(P)$ the flow generated by the (real) vector field $A$, and $A^{\theta}=\cos (\theta) A+\sin (\theta) J(A)$. The following lemma is proved in [6] for domains in $\mathbb{C}^{2}$.

Lemma 3. Let $\Omega$ be a smooth bounded pseudoconvex domain in $\mathbb{C}^{n}, n \geq 2$, and $P \in b \Omega$. Assume that the Levi form of $b \Omega$ has one degenerate eigenvalue at $P$. If there exist a neighborhood $U$ of $P$ in $b \Omega$, a smooth complex tangential (real) vector field $A$ defined on $U$, and $t_{0}>0$ such that $L(A-i J(A))=0$ at all points of the real two-dimensional manifold $F_{A^{\theta}}^{t}(P), 0 \leq t \leq t_{0}, 0 \leq \theta \leq 2 \pi$, then this manifold is an analytic disc, possibly after shrinking $t_{0}$. 
We want to show that the real two-dimensional manifold $M=\left\{F_{A^{\theta}}^{t}(p): 0 \leq t \leq\right.$ $\left.\widehat{t_{0}}, 0 \leq \theta \leq 2 \pi\right\}$ is actually a one dimensional complex manifold for some $\widehat{t_{0}}>0$. We follow [6] and indicate the necessary modifications. It suffices to show that the tangent space of $M$ is spanned by $A$ and $J(A)$. This means that it is invariant under $J$, and therefore $M$ is indeed a complex manifold. If we view $\theta$ as a parameter in the initial value problem that determines the flow generated by $A^{\theta}$ and set $f(t, \theta):=F_{A^{\theta}}^{t}(p)$, we see that $\frac{\partial f}{\partial \theta}$ is a solution of the following initial value problem:

$$
\left\{\begin{array}{l}
\left(\frac{\partial f}{\partial \theta}\right)^{\prime}=\frac{\partial A^{\theta}}{\partial x} \frac{\partial f}{\partial \theta}+\frac{\partial A^{\theta}}{\partial \theta} \\
\frac{\partial f}{\partial \theta}(0)=0
\end{array}\right.
$$

where $\frac{\partial A^{\theta}}{\partial x}$ is the Jacobian of $A^{\theta}$. We will find a solution to (11) in the form $a(t) A+$ $b(t) J(A)$. Then $\frac{\partial f}{\partial \theta}$, the unique solution of (1), is of this form. Therefore, both $\frac{\partial f}{\partial t}$ and $\frac{\partial f}{\partial \theta}$ are linear combinations of $A$ and $J(A)$. This will complete the proof of Lemma 3

If we substitute $a(t) A+b(t) J(A)$ for $\frac{\partial f}{\partial \theta}$ into (11), we get:

$$
\begin{aligned}
0= & a^{\prime}(t) A+(a(t) \sin (\theta)-b(t) \cos (\theta))[J(A), A] \\
& +b^{\prime}(t) J(A)+\sin (\theta) A-\cos (\theta) J(A) \\
0= & a(0)=b(0)
\end{aligned}
$$

Suppose that we can show that $[J(A), A]$ is a linear combination of $A$ and $J(A)$ at points of $M$. Note that the coefficients are automatically smooth. Then after collecting terms containing $A$ and $J(A)$, respectively, (2) becomes an initial value problem for $(a(t), b(t))$ that has a (unique) solution, and we are done. It is clear that $[J(A), A]$ is complex tangential (at points of $M$ ), because $A-i J(A)$ is a Levi null direction. In $\mathbb{C}^{2}$, this means that it is a (real) linear combination of $A$ and $J(A)$. The argument so far comes entirely from [6]. In higher dimensions, some additional work is needed.

The hypothesis that the Levi form has one degenerate eigenvalue at $P$ (hence at most one near $P$ ) implies that it is diagonalizable near $P$ (see e.g. [25], Lemma 2.1). Let $X_{1}, X_{2}, \cdots, X_{n-1}$ be smooth complex tangential vector fields of type $(1,0)$ that diagonalize the Levi form near $P$. We may assume that $X_{1}=A-i J(A)$ on $M$ (near $P$ ), since $A-i J(A)$ is an eigenvector associated with the eigenvalue 0 at points of $M$. Then the flows $F_{B^{\theta}}^{t}(P)$ associated with the vector fields $B^{\theta}$, where $B=\operatorname{Re} X_{1}$, also generate $M$, and we may therefore assume that $A-i J(A)=X_{1}$ in a full neighborhood of $P$ in the boundary. (In the proof of Proposition 1 below, we will actually be in this situation from the outset.) Thus the commutator we are interested in is $\left[X_{1}, \overline{X_{1}}\right]=2 i[B, J(B)]$. If $X_{1}$ were a Levi null field in a full neighborhood of $P$, then $\left[X_{1}, \overline{X_{1}}\right]$ would likewise be $\left([15)\right.$. However, we only know that $L\left(X_{1}, \overline{X_{1}}\right)=0$ on $M$. What we can assert is that $\left[X_{1}, \overline{X_{1}}\right]=Y-\bar{Y}+\varphi\left(L_{n}-\overline{L_{n}}\right)$, where $Y$ is a smooth complex tangential field of type $(1,0), L_{n}$ is the complex normal to the boundary, and $\varphi$ is a smooth, nonnegative function that vanishes on $M$. The nonnegativity of $\varphi$ is a consequence of the pseudoconvexity of $\Omega$. What we need is that on $M, Y$ is a multiple of $X_{1}$. The Jacobi identity for $X_{1}, \overline{X_{1}}$, and $X_{k}$ gives

$$
\left[Y-\bar{Y}+\varphi\left(L_{n}-\overline{L_{n}}\right), X_{k}\right]+\left[\left[\bar{X}_{1}, X_{k}\right], X_{1}\right]+\left[\left[X_{k}, X_{1}\right], \bar{X}_{1}\right]=0, k=1, \cdots, n-1 ;
$$


we have replaced $\left[X_{1}, \overline{X_{1}}\right]$ in the first term by $Y-\bar{Y}+\varphi\left(L_{n}-\overline{L_{n}}\right)$. The second and third commutators are complex tangential at points of $M$ if $k \geq 2$. To see this, note that $X_{1}$ is in the nullspace of the Levi form at points of $M$ and that both the commutators $\left[X_{k}, X_{1}\right]$ and $\left[X_{1}, X_{k}\right]$ are complex tangential in a full neighborhood of $P$ in $b \Omega$ (the latter because $X_{1}, \cdots, X_{n-1}$ diagonalize the Levi form near $P$ ). Consequently, the first commutator is complex tangential at points of $M$ as well. In addition, because $\varphi$ and its first order derivatives vanish at points of $M,\left[\varphi\left(L_{n}-\overline{L_{n}}\right), X_{k}\right]$ is zero at points of $M$. Therefore, $\left[Y-\bar{Y}, X_{k}\right]$ is complex tangential at points of $M$, and hence so is $\left[\bar{Y}, X_{k}\right]$. It follows that at points of $M, Y$ is in the nullspace of the Levi form and thus is a multiple of $X_{1}$. This completes the proof of Lemma 3. We remark that a similar use of the Jacobi identity occurs in 15. s

Once Lemma 3 is in hand, the proof of Proposition 1] can be completed as in [6], with only small modifications. Assume there is a compact subset $K$ of $\bar{\Omega}$ and a boundary point $P \in \widehat{K} \backslash K$ and there is no analytic disc in the boundary through $P$. We may assume that zero is an eigenvalue (with multiplicity one) of the Levi form at $P$. Take $V$ to be a neighborhood of $P$ small enough so that $V \cap K=\emptyset$ and so that there are complex tangential fields $X_{1}, \cdots, X_{n-1}$ of type $(1,0)$ which diagonalize the Levi form in a neighorhood of $\overline{b \Omega \cap V}$, with $L\left(X_{1}, \overline{X_{1}}\right)(P)=0$. By Lemma 3, there exist $\theta$ and $t_{0}>0$ such that $F_{A^{\theta}}^{t_{0}}(P) \in V$ and $F_{A^{\theta}}^{t_{0}}(P)$ is a strongly pseudoconvex point, where $A=X_{1}+\overline{X_{1}}$. Near $P$, choose a boundary coordinate system $\left(t_{1}, t_{2}, \cdots, t_{2 n-1}, r\right)$ such that $A^{\theta}=\frac{\partial}{\partial t_{1}}$, and $r$ is a defining function for $\Omega$. $V$ can be assumed to be contained in this coordinate patch. We will follow [6] to show that the integral curve of $A^{\theta}$ from $t_{1}=0$ to $t_{1}=t_{0}$, and hence $P$, can be separated by the level set of a strictly plurisubharmonic function from any compact subset of $\bar{\Omega} \backslash V$. This contradiction (to $P \in \widehat{K} \backslash K$ for some compact $K$ ) will complete the proof. As in [6], consider the auxiliary function $g\left(t_{1}, t_{2}, \cdots, t_{2 n-1}, r\right)=\left(t_{1}+\frac{1}{m}\right) /\left(1+m^{2}\left(t_{2}^{2}+\cdots+t_{2 n-1}^{2}\right)\right)$ and the sets $S_{c}=\left\{\left(t_{1}, t_{2}, \cdots, t_{2 n-1}, 0\right): g\left(t_{1}, t_{2}, \cdots, t_{2 n-1}, 0\right)=c, 0 \leq t_{1} \leq t_{0}\right\}$. Choose $m$ sufficiently large such that $S_{c} \subset \subset V \cap b \Omega$ for all $c$ with $1 / m \leq c \leq t_{0}+1 / m$. By shrinking $V$ if necessary we may assume that any point in the set $\{z \in b \Omega \cap V$ : $\left.t_{1}(z) \geq t_{0}\right\}$ is strongly pseudoconvex. In [6], $g$ is modified, but the modification is specified to be of order $r^{2}$. Here, it is important to also have a term that is of order $r$. Specifically, set $h=g+\mu r+\nu r^{2}$ for $\mu, \nu$ positive numbers to be determined. We have for $\tau>0$ and $W=\sum_{j=1}^{n} w_{j} \partial / \partial z_{j}$ (at boundary points, where $r=0$ ) $L_{e^{\tau h}}(W, \bar{W})=\tau e^{\tau h}\left(L_{g}(W, \bar{W})+\mu L_{r}(W, \bar{W})+\tau|W(g)+\mu W(r)|^{2}+2 \nu|W(r)|^{2}\right)$. Set $\left.X_{n}=\left(1 / \sum_{j=1}^{n}\left|\partial r / \partial z_{j}\right|^{2}\right) \sum_{j=1}^{n}\left(\partial r / \partial \overline{z_{j}}\right) \partial / \partial z_{j}\right)$ (so that $\left.X_{n}(r)=1\right)$. We express $W$ in terms of the basis $\left\{X_{1}, X_{2}, \cdots, X_{n-1}, X_{n}\right\}$ as $W=\sum_{j=1}^{n} \alpha_{j} X_{j}$, and apply the inequalities $2 a b \leq \varepsilon a^{2}+(1 / \varepsilon) b^{2}$ for $\varepsilon>0,|a+b|^{2} \geq \frac{1}{2}|a|^{2}-|b|^{2}$, and $\left|\sum_{j=1}^{n} a_{j}\right|^{2} \leq$ $n \sum_{j=1}^{n}\left|a_{j}\right|^{2}$, to obtain

$$
\begin{aligned}
\frac{1}{\tau} e^{-\tau h} L_{e^{\tau h}}(W, \bar{W}) & \geq \frac{\tau}{2}\left|X_{1}(g)\right|^{2}\left|\alpha_{1}\right|^{2}+\sum_{j=2}^{n-1}\left(\mu L_{r}\left(X_{j}, \overline{X_{j}}\right)-\tau n\left|X_{j}(g)\right|^{2}\right)\left|\alpha_{j}\right|^{2} \\
& +2 \nu\left|\alpha_{n}\right|^{2}-\left(\tau n\left|X_{n}(g)\right|^{2}+\tau n \mu^{2}+\mu\left|L_{r}\left(X_{n}, \overline{X_{n}}\right)\right|\right)\left|\alpha_{n}\right|^{2} \\
& - \text { s.c. } \sum_{j=1}^{n}\left|\alpha_{j}\right|^{2}-\text { l.c. }(\mu)\left|\alpha_{n}\right|^{2}+L_{g}(W, \bar{W}),
\end{aligned}
$$


where s.c. denotes a small constant, and l.c. $(\mu)$ denotes a large constant whose size depends on $\mu$. Note that on $\bar{V} \cap b \Omega,\left|X_{1}(g)\right| \approx|A(g)|+|J(A)(g)| \geq\left|A^{\theta}(g)\right|=$ $\left|\partial g / \partial t_{1}\right|>0$ and $L_{r}\left(X_{j}, \overline{X_{j}}\right)>0$ for $2 \leq j \leq n-1$. Therefore, we can first choose $\tau$, then $\mu$, and then $\nu$, big enough so that $L_{e^{\tau h}}(W, \bar{W}) \geq|W|^{2}$ on $\bar{V} \cap b \Omega$. So $e^{\tau h}$ is strictly plurisubharmonic near $\bar{V} \cap b \Omega$. From here on, the argument is exactly as in 6], p. 54-55; we only sketch it. Choose a smooth function $\psi_{s}\left(t_{1}\right)$ which is identically 1 for $t_{1} \leq t_{0}$, and identically 0 for $t_{1} \geq s$, where $s>t_{0}$ is chosen small enough so that the level sets $\left\{\psi_{s} e^{\tau h}=c\right\}$ are contained in $V$ for $1 / m \leq c \leq t_{0}+1 / m$. To deal with the direction transverse to the boundary, Catlin applies his construction in [6], Theorem 3.1.6 (see also [7], Proposition 3.1.6). In our situation, this construction yields a strictly plurisubharmonic function on a neighborhood $V_{1}$ of $P$ with $\left\{F_{A^{\theta}}^{t}(P)\right.$ : $\left.0 \leq t \leq t_{0}\right\} \subset \subset V_{1} \subset \subset V$, whose superlevel set determined by $P$ is a compact subset of $V_{1}$. Composition with a suitable convex increasing function finally results in a plurisubharmonic function defined on all of $\bar{\Omega}$ (by extension by 0 ) that separates $P$ from any compact subset of $\bar{\Omega} \backslash V$. This completes the proof of (the nontrivial direction of) Proposition 1 .

\section{REFERENCES}

1. E. Bedford and J. E. Fornaess, Complex manifolds in pseudoconvex boundaries, Duke Math.J. 48, Nr. 1(1981),179-288.

2. S. Bell, Differentiability of the Bergman kernel and pseudo-local estimates, Math. Z. 192 (1986), 467-472.

3. H. P. Boas, Extension of Kerzman's theorem on differentiability of the Bergman kernel function, Indiana Univ. Math. J. 36, Nr. 3 (1987), 495-499.

4. H. P. Boas and E. J. Straube, De Rham cohomology of manifolds containing the points of infinite type, and Sobolev estimates for the $\bar{\partial}$-Neumann problem, J. Geometric Analysis 3, Nr.3 (1993), $225-235$.

5. _- Global regularity of the $\bar{\partial}-$ Neumann problem: a survey of the $L_{2}$-Sobolev theory, Several Complex Variables, M. Schneider and Y.-T. Siu, eds., MSRI Publications 37, Cambridge University Press, 1999.

6. D. W. Catlin, Boundary behavior of holomorphic functions on weakly pseudoconvex domains, Princeton University Ph.D. Thesis 1978.

7. __ Boundary behavior of holomorphic functions on weakly pseudoconvex domains, J. Differ. Geom. 15 (1980), 605-625.

8. __ Necessary conditions for subellipticity and hypoellipticity for the $\bar{\partial}$-Neumann problem on pseudoconvex domains, Recent Developments in Several Complex Variables, J. E. Fornaess ed., Annals of Math. Studies 100, Princeton Univ. Press 1981, 93-100.

9. _ Global regularity of the $\bar{\partial}-$ Neumann problem, Complex Analysis of Several Variables, Y.-T. Siu ed., Proc. Symp. Pure Math. 41, 1984, 39-49.

10. S.-C. Chen and M.-C. Shaw, Partial Differential Equations in Several Complex Variables, Studies in Advanced Mathematics, American Mathematical Society/International Press, 2001.

11. M. Christ, Remarks on global irregularity in the $\bar{\partial}-$ Neumann problem, Several Complex Variables, M. Schneider and Y.-T. Siu, eds., MSRI Publications 37, Cambridge University Press, 1999.

12. M. Christ and S. Fu, Compactness in the $\bar{\partial}-$ Neumann problem,magnetic Schrödinger operators, and the Aharonov-Bohm effect, Advances in Mathematics, in press

13. K. Diederich and P. Pflug, Necessary conditions for hypoellipticity of the $\bar{\partial}$-problem, Recent Developments in Several Complex Variables, J. E. Fornaess ed., Annals of Math. Studies 100, Princeton Univ. Press 1981, 151-154. 
14. G. B. Folland and J. J. Kohn, The Neumann Problem for the Cauchy-Riemann Complex, Annals of Mathematics Studies 75, Princeton University Press, 1972.

15. M. Freeman, The Levi form and local complex foliations, Proc. Amer. Math. Soc. 57 (1976), no:2, 369-370.

16. S. Fu and E. J. Straube, Compactness of $\bar{\partial}-$ Neumann problem on convex domains J. Func. Analysis 159 (1998), 629-641

17. Compactness of the $\bar{\partial}$-Neumann problem, Complex Analysis and Geometry, J. McNeal ed., Ohio State Univ. Math. Res. Inst. Publ., 9, de Gruyter, Berlin, 2001, 141-160

18. Semi-classical analysis of Schrödinger operators and compactness in the $\bar{\partial}-N e u m a n n$ problem, Journal of Math. Analysis and Application 271, 2002, pp. 267-282, correction in Journal of Math. Analysis and Applications 280, 2003, 195-196.

19. M. Hakim and N. Sibony, Spectre de $A(\bar{D})$ pour les domaines faiblement pseudoconvexes, J. Funct. Anal. 37 (1980), 127-135.

20. P. S. Harrington, Compactness and subellipticity for the $\bar{\partial}-$ Neumann operator on domains with minimal smoothness, University of Notre Dame Ph.D. Thesis, 2004.

21. T. Hefer and I. Lieb, On the compactness of the $\bar{\partial}-$ Neumann operator, Ann. Fac. Sci. Toulouse Math. (6), 9 (2000), 415-432.

22. L. Hörmander, $L^{2}$ estimates and existence theorems for the $\bar{\partial}$ operator, Acta Math. 113 (1965), 89-152

23. M. Kim, Inheritance of noncompactness of the $\bar{\partial}-$ Neumann problem, J. Math. Anal. and Appl. 302 (2005), 450-456.

24. J. J. Kohn and L. Nirenberg, Non-coercive boundary value problems, Comm. Pure Appl. Math. 18 (1965), 443-492.

25. M. Machedon, Szegö kernels on pseudoconvex domains with one degenerate eigenvalue, Ann. of Math. 128 (1988), 619-640.

26. P. Matheos, A Hartogs domain with no analytic discs in the boundary for which the $\bar{\partial}-N e u m a n n$ problem is not compact, University of California Los Angeles Ph.D. Thesis, 1997.

27. J. D. McNeal, A sufficient condition for compactness of the $\bar{\partial}-$ Neumann problem, Journal of Functional Analysis 195, 2002, 190-205.

28. T. Ohsawa and K. Takegoshi, On the extension of $L^{2}$ holomorphic functions, Math Z. 195 (2) (1987), 197-204.

29. R. M. Range, Holomorphic Functions and Integral Representations in Several Complex Variables, Graduate Texts in Mathematics 108, Springer, 1986

30. N. Sibony, Hypoellipticité pour l'opérateur $\bar{\partial}$, Math. Ann. 276 (1987), no. 2, 279-290.

31. Une classe de domaines pseudoconvexes, Duke Math. Journal 55, 1987, 299-319.

32. E. J. Straube, Geometric conditions which imply compactness of the $\bar{\partial}$-Neumann operator, Ann. Inst. Fourier 54, fasc. 3 (2004), 699-710.

33. E. J. Straube and M. K. Sucheston, Plurisubharmonic defining functions, good vector fields, and exactness of a certain one form, Monatshefte für Mathematik 136 (2002), 249-258.

34. K. Takegoshi, A new method to introduce a priori estimates for the $\bar{\partial}-$ Neumann problem, Complex Analysis, K. Diederich ed., Aspects of Mathematics E17, Vieweg 1991.

E-mail address, Sönmez Şahutoğlu: sahutogl@math.tamu.edu

E-mail address, Emil J. Straube: straube@math.tamu.edu

Department of Mathematics, Texas A\&M University, College Station, TX 778433368 